\title{
RESISTÊNCIA DA LINHA DE COLA DE PAINÉIS DE Pinus taeda COLADOS LATERALMENTE COM DIFERENTES ADESIVOS
}

\author{
Merielen de Carvalho Lopes ${ }^{1 *}$, Graciela Inês Bolzon de Muniz², Jorge Luis Monteiro de Matos², \\ Valcineide Oliveira de Andrade Tanobe ${ }^{2}$, Carlos Augusto Fernandes Chinasso ${ }^{3}$, Silviana Rosso ${ }^{4}$
}

*Autora para correspondência: merielen-lopes@hotmail.com

RESUMO: No presente estudo, objetivou-se comparar a resistência da linha de cola de painéis de Pinus taeda colados lateralmente com diferentes adesivos industriais. Foram analisados três tipos de adesivos (poli(vinil) acetato (PVA $)$, emulsão polimérica de isocianato (EPI) e poliuretano (PU)) colados em duas orientações (radial e tangencial) e avaliados após os condicionamentos de ensaio (a seco e a úmido). Foram preparados 360 corpos de prova para a determinação da resistência ao cisalhamento dos painéis colados lateralmente de Pinus taeda e, estes foram testados em máquina universal de ensaio EMIC. A resistência da linha de cola ao cisalhamento a seco foi inferior nas peças coladas no sentido tangencial para os três tipos de adesivos analisados. $\mathrm{O}$ adesivo PU apresentou os maiores valores médios de resistência ao cisalhamento nos condicionamentos aplicados, diferindo, estatisticamente, do adesivo PVA e do adesivo EPI quando testado a seco e não diferindo dos demais adesivos quando testado a úmido. O maior valor percentual de falha na madeira foi observado após o ensaio de resistência ao cisalhamento a seco para o adesivo EPI colado no sentido radial. E em cisalhamento a úmido, o adesivo EPI e o adesivo PU colados no sentido tangencial apresentaram valores médios superiores de falha na madeira. Assim, foi possível concluir que a resistência da linha de cola da madeira de Pinus taeda foi influenciada pelo tipo de adesivo e pela orientação de colagem, além de se comportar diferenciadamente conforme a condição de uso (seco ou úmido).

Palavras-chave: Painel sarrafeado, madeira, colagem.

\section{STRENGHT OF THE GLUE LINE OF EDGE GLUED PANELS OF Pinus taeda MADE WITH DIFFERENT ADHESIVES}

\begin{abstract}
The study compares the strength of the glue line of edge glued panels of Pinus taeda made with different industrial adhesives. Three types of adhesives (poly (vinyl) acetate (PVAc), emulsion polymer isocyanate (EPI) and polyurethane (PU)) glued in two side gluing orientation (radial and tangential) and analyzed after the conditioning tests (dry and wet) were analyzed. The 360 specimens were prepared for determining the shear strength of edge glued panels of Pinus taeda, and these were tested in a universal testing machine called EMIC. The shear strength of the glue line at dry condition was lower in the tangential side for the three types of adhesives analyzed. The PU adhesive showed the highest values of shear strength in the applied conditions, differing from PVAc and EPI adhesive when tested on dry condition and did not differ from the others adhesives when tested on wet condition. The highest percentage of wood failure was observed after the shear strength test at dry condition to EPI adhesive glued in the radial side. And in the wet condition, the EPI adhesive and PU adhesive glued in tangential side showed higher mean values of wood failure. Thus, it was possible to conclude that the strength of the glue line of Pinus taeda wood was influenced by the type of adhesive and side gluing orientation, and showed differently behavior according to the condition of use (wet or dry).
\end{abstract}

Key words: Slatted panel, wood, bonding.

\section{INTRODUÇÃO}

O setor florestal no Brasil destaca-se pela produção e exportação de produtos de maior valor agregado a partir da madeira de reflorestamento, como portas, molduras, painel colado lateralmente (EGP), pisos de madeira e outros componentes estruturais (ASSOCIAÇÃO BRASILEIRA DA INDÚSTRIA DE MADEIRA MECANICAMENTE PROCESSADA - ABIMCI, 2007).

Segundo Carneiro et al. (2001), mais de $70 \%$ dos produtos derivados da madeira consomem algum tipo de

${ }^{1}$ Universidade Federal de Pelotas - Pelotas, Rio Grande do Sul, Brasil

${ }^{2}$ Universidade Federal do Paraná - Curitiba, Paraná, Brasil

${ }^{3}$ Fundação do Meio Ambiente - Blumenau, Santa Catarina, Brasil

${ }^{4}$ Universidade Federal do Pampa - São Gabriel, Rio Grande do Sul, Brasil adesivo, em razão da crescente substituição da madeira por seus derivados, gerando o crescimento no consumo de adesivos sintéticos. Dentre os adesivos sintéticos utilizados pela indústria brasileira da madeira e móveis, destaca-se o consumo dos adesivos base poli(vinil) acetato ou PVA, vulgarmente conhecidos como "cola branca' e, mais recentemente, o consumo de adesivos base isocianatos ou adesivos poliuretanos (PU).

A emulsão de PVA $_{c}$ é formada pela polimerização em água de monômeros de vinil, predominantemente monômero de vinil acetato (VAM). Os adesivos de poli

Cerne, Lavras, v. 19, n. 4, p. 613-619, out./dez. 2013 
(acetato de vinila) são produzidos em duas etapas: a primeira consiste em uma reação de oxidação entre etileno e ácido acético para produzir o acetato de vinil, produto líquido transparente e muito inflamável; na segunda etapa, o acetato de vinil é polimerizado em uma emulsão aquosa formando uma dispersão aquosa de poli (acetato de vinila) (BANDEL, 1991).

Os adesivos líquidos poliuretânicos, com $100 \%$ de sólidos, apresentam excelentes propriedades de adesão (pela sua natureza polar), excelente resistência química (quando reticulado), flexibilidade, desempenho bom em baixas temperaturas e podem ser curados lenta ou rapidamente. Algumas das desvantagens são: a limitada estabilidade térmica, manuseio, alto custo e necessidade de mistura (FRIHART, 2005).

O polímero de difenilmetano-diisocianato (MDI) pode ser emulsificado em água, formando uma resina chamada emulsão polimérica de isocianato - EPI (CONNER, 2001), que deve ser ativada pelo uso de um endurecedor ou catalisador. Logo, emulsões poliméricas de isocianato são adesivos bi-componentes, que devem ser misturados antes da aplicação. Em razão da elevada reatividade, o tempo de aplicação deve ser ajustado de acordo com a recomendação do fabricante. Entretanto, podem ser utilizadas em equipamentos tradicionais para colagem de madeira. O uso comercial compreende a colagem de painéis, a colagem de plásticos à superfície da madeira e a colagem da alma de OSB na estrutura da flange de uma viga tipo I.

Bustos et al. (2003) citam que os adesivos à base isocianatos como as poliuretanas estão ganhando aceitação na América do Norte para uma variedade de aplicações estruturais e não-estruturais. Os adesivos poliuretanos reativos (PUR) oferecem características interessantes, como elevada resistência da linha de cola e a cura pela exposição à temperatura ambiente. As colagens obtidas com esses adesivos apresentam resistência à fadiga, à umidade e a tratamentos de exposição ao calor (PAGEL; LUCKMAN, 1984).

Segundo Frihart (2005), a resistência do adesivo é definida mecanicamente como a força necessária para separar os substratos que estão colados. A resistência mecânica é dependente das ligações químicas primárias e secundárias da cadeia do polímero do adesivo, da madeira e da interface madeira-adesivo.

As tensões geradas pela interação entre a madeira e o adesivo são de suma importância no balanço geral da resistência do painel. Quanto maior a resistência da linha de cola em relação à resistência da madeira, maior será a percentagem de falhas da madeira na interface com o adesivo (IWAKIRI et al., 2005).

O tipo de adesivo, o tempo de cura e a pressão de aplicação têm grande influência no comportamento de resistência das emendas (BUSTOS et al., 2003).

Ligações de madeira com alta resistência resultam quando adesivos poliuretanos reativos são usados e quando a superfície foi preparada cuidadosamente antes da colagem (KOLLMANN et al., 1975).

Os estudos de Özçifçi e Yapici (2007) demonstraram que a resistência da colagem é dependente do tipo de preparação da superfície, da direção de corte (radial ou tangencial), dos adesivos e das espécies de madeira. E avaliando diferentes tipos de adesivos, encontraram maior resistência da colagem para o adesivo $\mathrm{PVA}_{\mathrm{c}}$ e menor para 0 adesivo PU (D-VTKA).

Vital et al. (2006), estudando a qualidade de juntas coladas com lâminas de madeira oriundas de três regiões do tronco de Eucalyptus grandis, Eucalyptus saligna e Pinus elliottii, pela análise estatística da resistência ao cisalhamento, encontraram diferenças significativas entre espécies, tipo de adesivo e combinações de lâminas e, observaram ainda uma interação significativa entre espécie e tipo de adesivo.

Uysal (2005), estudando a resistência da colagem e a estabilidade dimensional entre diferentes tipos de adesivos e espécies de madeira, observou os melhores resultados para a madeira de Pinus sylvestris e adesivo uréia-formaldeido (UF) e adesivo poliuretano (D-VTKA).

Fotsing e Alexis (2003), avaliando a resistência de adesivos poli (acetato de vinila), comercializados no mercado da madeira de Cameron, encontraram valores médios de resistência da linha de cola, variando entre 4,9 a $15,4 \mathrm{kN}$, conforme o produto avaliado.

Neste trabalho, objetivou-se comparar a resistência da linha de cola de painéis de Pinus taeda colados lateralmente com diferentes tipos de adesivos utilizados pela indústria brasileira, pela análise destrutiva das amostras.

\section{MATERIAL E MÉTODOS}

Foram usados três tipos de adesivos, sendo um adesivo poli(vinil) acetato (PVA ), um adesivo base emulsão polimérica de isocianato (EPI), devidamente catalisado, e um adesivo poliuretano (PU), monocomponente, comercialmente fornecidos e utilizados no segmento da madeira e móveis do Brasil.

Cerne, Lavras, v. 19, n. 4, p. 613-619, out./dez. 2013 
Os painéis colados lateralmente em madeira de Pinus taeda foram produzidos no Laboratório de Aplicações para a Madeira, da empresa National Starch \& Chemical Industrial Ltda, utilizando-se variáveis de processo controladas, sendo: os sarrafos de madeira a serem colados classificados no sentido radial e tangencial; a superfície dos sarrafos a ser colada foi preparada em lixadeira TECMATIC, com grã 50; a gramatura média aplicada foi de $200 \mathrm{~g} / \mathrm{m}^{2}$; a pressão de colagem foi de 07 $\mathrm{kgf} / \mathrm{cm}^{2}$; como tempo de montagem foi considerado a somatória do tempo gasto desde a aplicação do adesivo na superfície das peças até o fechamento da prensa, sendo que, para o adesivo $\mathrm{PVA}_{\mathrm{c}}$ foi igual a 4 minutos, para o adesivo EPI foi igual a 5 minutos e para o adesivo PU foi igual a 3 minutos; o tempo de prensagem para o adesivo $\mathrm{PVA}_{\mathrm{c}}$ foi igual a 3 minutos, por meio do uso de uma prensa de alta freqüência Maquimóvel PAF 1200, para o adesivo EPI foi igual a 30 minutos e para o adesivo PU foi igual a 15 minutos, conforme as recomendações do fabricante, sendo utilizada uma prensa fria tipo carrossel Metalúrgica América.

Os corpos de prova, com dimensões de 10 x 20 x $150 \mathrm{~mm}$, foram dimensionados de acordo com a norma européia EN 205 (EUROPEAN STANDARD - EN, 2002), aplicada à determinação da resistência de adesivos de uso não estrutural. Foram preparados 360 corpos de prova (cps) para a determinação da resistência ao cisalhamento da linha de cola, sendo $120 \mathrm{cps}$ para cada tipo de adesivo (PVA, EPI e PU), divididos em $30 \mathrm{cps}$ para cada orientação de colagem (radial e tangencial) e 30 cps para cada condicionamento (à seco e à úmido), totalizando seis tratamentos em cada condicionamento. As etapas de condicionamento para a determinação da resistência ao cisalhamento foram baseadas na norma européia EN 204 (EN, 2001). Os corpos de prova foram tracionados em máquina universal de ensaios da marca EMIC, modelo DL 10000, com célula de carga de $500 \mathrm{kgf}$ e os dados de resistência ao cisalhamento foram computados pelo programa TESC, específico do equipamento de ensaio.

A classificação visual da região de cisalhamento da linha de cola foi aplicada como um método de verificação da falha na madeira. A falha na madeira foi classificada como um percentual variando de 0 a $100 \%$, onde: $0 \%$ representou uma região de cisalhamento na linha de cola sem qualquer ou inferior a $1 / 4$ de vestígios de fibra de madeira nas faces rompidas do corpo de prova; $25 \%$ representou uma região de cisalhamento na linha de cola igual a $1 \frac{1}{4}$ e inferior a $1 / 2$ de vestígios de fibra de madeira nas faces rompidas do corpo de prova; $50 \%$ representou uma região de cisalhamento na linha de cola igual a $1 \frac{1}{2}$ e inferior a $3 / 4$ de vestígios de fibra da madeira nas faces rompidas do corpo de prova; $75 \%$ representou uma região de cisalhamento da linha de cola iguais a $3 / 4$ e inferior a 1 de vestígios de fibra da madeira nas faces rompidas do corpo de prova e $100 \%$ representou uma região de cisalhamento na linha de cola integral da madeira, ou seja, observou-se apenas a presença da madeira nas faces rompidas do corpo de prova.

Por meio do programa estatístico Statgraphics Plus 5.1, foi possível realizar a análise estatística descritiva, a análise de variância e teste de comparação de médias para a resistência da linha de cola pelo método Least Significant Difference (LSD) e, a falha na madeira foi analisada por teste não paramétrico, pelo método de Kruskal Wallis, para os três tipos de adesivos e as duas orientações da peça, distintamente de acordo com o condicionamento aplicado.

\section{RESULTADOS E DISCUSSÃO}

Na Tabela 1, são apresentados os valores médios de resistência da linha de cola dos adesivos PVA $_{c}$, EPI e PU testados de acordo com o condicionamento aplicado (a seco e a úmido) e da orientação da peça (radial e tangencial).

A resistência média da linha de cola da madeira de Pinus taeda colada com adesivo PVA no sentido tangencial, em cisalhamento a seco, foi de 7,20 MPa, valor este inferior ao obtido por Özçifçi e Yapici (2007) para a resistência da colagem de Pinus sylvestris com o adesivo $\mathrm{PVA}_{c}$, na posição tangencial de 14,52 MPa. Já no sentido radial, o adesivo PVA $_{\mathrm{c}}$ apresentou resistência média ao cisalhamento a seco de 9,12 MPa e a úmido igual a 1,54 MPa, valor este inferior ao obtido no sentido tangencial $(1,91 \mathrm{MPa})$.

Os valores médios observados para o adesivo $\mathrm{PVA}_{c}$, em cisalhamento a seco, foram superiores aos valores médios observados por Vital et al. (2006) avaliando a resistência ao cisalhamento de dois adesivos comerciais de poliacetato de vinila, de média e alta viscosidade, aplicados a madeira de Pinus elliottii, encontraram valores de tensão de ruptura iguais a 5,41 MPa e 5,64 MPa, respectivamente.

Observou-se que a resistência média da linha de cola da madeira de Pinus taeda com adesivo PU, em cisalhamento a seco, no sentido radial foi igual a 9,61 MPa e no sentido tangencial de $8,43 \mathrm{MPa}$, valor este inferior ao obtido por Özçifçi e Yapici (2007) na análise da madeira de Pinus sylvestris na posição tangencial (11,71 MPa), porém superiores aos valores observados por Burdulu et al. (2006) para a madeira de Pinus brutia em ambas orientações de colagem.

Cerne, Lavras, v. 19, n. 4, p. 613-619, out./dez. 2013 
Tabela 1 - Valores médios da resistência da linha de cola dos adesivos analisados, em função do condicionamento aplicado e orientação da peça.

Table 1 -Average values of strength of the glue line from tested adhesives, according to applied conditioning and side orientation.

\begin{tabular}{|c|c|c|c|c|c|c|}
\hline $\begin{array}{l}\text { Condicionamento/ } \\
\text { Tratamentos }\end{array}$ & Freqüência & $\begin{array}{l}\text { Média* } \\
(\mathrm{MPa})\end{array}$ & Erro padrão & $\begin{array}{l}\mathrm{CV} \\
(\%)\end{array}$ & $\begin{array}{l}\text { Mínimo } \\
(\mathrm{MPa})\end{array}$ & $\begin{array}{c}\text { Máximo } \\
(\mathrm{MPa})\end{array}$ \\
\hline \multicolumn{7}{|l|}{ Seco } \\
\hline \multicolumn{7}{|l|}{ Radial } \\
\hline $\mathrm{PVA}_{\mathrm{c}}(1)$ & 30 & $9,12 \mathrm{~cd}$ & 0,314 & 16,6 & 6,30 & 12,20 \\
\hline EPI (2) & 30 & $8,67 \mathrm{c}$ & 0,314 & 15,4 & 6,26 & 10,75 \\
\hline PU (3) & 30 & $9,61 \mathrm{~d}$ & 0,314 & 17,8 & 5,98 & 12,74 \\
\hline \multicolumn{7}{|l|}{ Tangencial } \\
\hline $\mathrm{PVA}_{\mathrm{c}}$ (4) & 30 & $7,21 \mathrm{a}$ & 0,314 & 26,3 & 3,23 & 11,71 \\
\hline EPI (5) & 30 & $7,63 \mathrm{ab}$ & 0,314 & 25,2 & 4,54 & 12,61 \\
\hline PU (6) & 30 & $8,43 \mathrm{bc}$ & 0,314 & 22,3 & 2,65 & 11,23 \\
\hline \multicolumn{7}{|l|}{ Úmido } \\
\hline \multicolumn{7}{|l|}{ Radial } \\
\hline $\mathrm{PVA}_{\mathrm{c}}(1)$ & 30 & $1,54 \mathrm{a}$ & 0,127 & 30,1 & 0,70 & 2,54 \\
\hline EPI (2) & 30 & $3,57 \mathrm{~d}$ & 0,127 & 20,9 & 2,00 & 5,16 \\
\hline PU (3) & 30 & $3,50 \mathrm{~d}$ & 0,127 & 19,7 & 2,18 & 5,09 \\
\hline \multicolumn{7}{|l|}{ Tangencial } \\
\hline $\mathrm{PVA}_{\mathrm{c}}$ (4) & 30 & $1,91 \mathrm{~b}$ & 0,127 & 19,6 & 1,34 & 2,75 \\
\hline EPI (5) & 30 & $2,91 \mathrm{c}$ & 0,127 & 35,9 & 0,41 & 5,18 \\
\hline PU (6) & 30 & $4,09 \mathrm{e}$ & 0,127 & 15,9 & 2,82 & 5,24 \\
\hline
\end{tabular}

*Médias seguidas por mesma letra não diferem significativamente entre si pelo teste (LSD) ao nível de 5\% de significância.

Os baixos valores de resistência da linha de cola da madeira de Pinus taeda observados neste trabalho podem estar relacionados com a preparação da superfície de colagem utilizada, pois como citado por Mitchell e Lemaster (2002) baixos valores de resistência da linha de cola podem ser obtidos quando a superfície da madeira for preparada em lixadeira, o que segundo Özçifçi e Yapici (2007) se justifica pelo fato da lixadeira aumentar a rugosidade superficial da madeira, causando uma ligação mecânica e diminuindo a ligação química entre as superfícies.

De acordo com os resultados (Tabela 1), os menores valores médios da resistência ao cisalhamento a seco da madeira de Pinus taeda foram observados no sentido tangencial para os três tipos de adesivos estudados. Quando analisados os valores médios de resistência obtidos no sentido radial, apesar do adesivo PU ter apresentado o maior valor médio de resistência, este não diferiu estatisticamente do adesivo PVA, ou seja, a colagem no sentido radial para os adesivos $\mathrm{PVA}_{\mathrm{c}}$ e PU apresentaram o melhor desempenho mecânico a seco. Esse comportamento da resistência da linha de cola entre os adesivos $\mathrm{PVA}_{c} \mathrm{e}$ PU, no sentido radial, pode ser considerado equivalente ao observado por Burdulu et al. (2006) para a madeira de Pinus brutia.

No cisalhamento a úmido da madeira de Pinus taeda colada com adesivo $\mathrm{PVA}_{\mathrm{c}}$ apresentou os menores valores médios de resistência e, estes valores diferiram significativamente entre os sentidos radial e tangencial, assim quando a madeira for colada com adesivo PVA no sentido tangencial a linha de cola terá maior resistência mecânica. Quando colado com o adesivo EPI é possível afirmar que no sentido radial, a resistência da linha de cola da madeira de Pinus taeda não difere significativamente do adesivo PU, ou seja, os adesivos EPI e PU oferecem desempenho mecânico equivalentes a úmido quando colados na orientação radial. E, por fim, o adesivo PU colado no sentido tangencial apresentou a maior resistência da linha de cola em cisalhamento a úmido quando comparado aos demais adesivos e orientação radial.

Cerne, Lavras, v. 19, n. 4, p. 613-619, out./dez. 2013 
Por meio da análise de variância multifatorial (Tabela 2), observou-se diferença significativa da resistência ao cisalhamento a seco dos fatores adesivo (PVA, EPI e PU) e orientação de colagem (radial e tangencial), porém com interação não significativa entre esses fatores. Já, a resistência ao cisalhamento a úmido apresentou diferença significativa do fator adesivo e não significativa do fator orientação de colagem, ou seja, quando analisados como efeito, o sentido radial ou tangencial não influenciaram significativamente nos valores médios de resistência observados. Porém, observou-se interação significativa entre os fatores adesivo e orientação de colagem com a resistência ao cisalhamento a úmido, ou seja, o comportamento mecânico da linha de cola úmida é afetado pela combinação entre os fatores.

Tabela 2 - Valores médios e interação dos fatores adesivo e orientação da peça, conforme o condicionamento aplicado.

Table 2 - Average values and interaction of adhesive and side orientation factors, according to the applied conditioning.

\begin{tabular}{lccccc}
\hline \multirow{2}{*}{ Fator } & & \multicolumn{4}{c}{$\begin{array}{c}\text { Resistência ao cisalhamento } \\
\text { médio* }\end{array}$} \\
\cline { 3 - 7 } & & Seco & P-valor & Úmido & P-valor \\
\cline { 3 - 7 } Adesivo & PVA & $8,17 \mathrm{a}$ & & $1,73 \mathrm{a}$ & \\
(A) & EPI & $8,15 \mathrm{a}$ & 0,0076 & $3,24 \mathrm{~b}$ & 0,0000 \\
& PU & $9,02 \mathrm{~b}$ & & $3,80 \mathrm{c}$ & \\
\hline Orientação & Radial & $9,14 \mathrm{a}$ & 0,0000 & $2,87 \mathrm{a}$ & 0,3314 \\
(B) & Tangencial & $7,76 \mathrm{~b}$ & & $2,97 \mathrm{a}$ & \\
\hline Interação AB & & - & 0,3377 & - & 0,0000 \\
\hline
\end{tabular}

*Médias seguidas por mesma letra não diferem significativamente entre si pelo teste (LSD) ao nível de 5\% de significância.

Os maiores valores de falha na madeira (Tabela 3 ) foram obtidos após o cisalhamento a seco da linha de cola da madeira de Pinus taeda para o adesivo EPI colado no sentido radial e, após o cisalhamento a úmido para o adesivo EPI colado no sentido tangencial.

Por meio da análise estatística da falha na madeira, após o ensaio de resistência da linha de cola observou-se uma alta variabilidade dos dados, esta pode ser atribuída à preparação da linha de cola, à baixa ligação mecânica e química entre o adesivo e a madeira e a subjetividade do avaliador na análise, e também a não normalidade do conjunto de dados, sendo testada e rejeitada a hipótese de nulidade do desvio padrão da média igual para todos os tratamentos aplicados ( 3 tipos de adesivos e 2 orientações da peça). Assim, optou-se pela aplicação do teste não
Tabela 3 - Valores médios e classificação das médias da falha na madeira dos adesivos analisados, em função do condicionamento aplicado e orientação da peça.

Table 3 - Average values and average rank of wood failure for tested adhesives, according to the applied conditioning and the side orientation.

\begin{tabular}{|c|c|c|c|c|c|}
\hline $\begin{array}{l}\text { Condicionamento/ } \\
\text { Tratamentos }\end{array}$ & $\begin{array}{c}\text { Fre- } \\
\text { qüência }\end{array}$ & $\begin{array}{l}\text { Média } \\
(\%)\end{array}$ & $\begin{array}{c}\text { Erro } \\
\text { padrão }\end{array}$ & $\begin{array}{l}\text { Desvio } \\
\text { padrão }\end{array}$ & $\begin{array}{l}\text { Classifi- } \\
\text { cação* }^{*}\end{array}$ \\
\hline \multicolumn{6}{|l|}{ Seco } \\
\hline \multicolumn{6}{|l|}{ Radial } \\
\hline $\mathrm{PVA}_{c}(1)$ & 30 & 49,33 & 6,788 & 41,612 & $94,03 \mathrm{a}$ \\
\hline EPI (2) & 30 & 81,33 & 6,788 & 24,030 & $128,75 \mathrm{~b}$ \\
\hline PU (3) & 30 & 39,33 & 6,788 & 41,827 & $75,58 \mathrm{a}$ \\
\hline \multicolumn{6}{|l|}{ Tangencial } \\
\hline $\mathrm{PVA}_{\mathrm{c}}$ (4) & 30 & 38,00 & 6,788 & 33,104 & 70,61 a \\
\hline EPI (5) & 30 & 45,10 & 6,788 & 40,890 & $81,68 \mathrm{a}$ \\
\hline PU (6) & 30 & 52,33 & 6,788 & 38.812 & $92,33 \mathrm{a}$ \\
\hline $\mathrm{T}_{\text {calculado }}$ & & & & & 24,4335 \\
\hline P-valor & & & & & 0,00017 \\
\hline \multicolumn{6}{|l|}{ Úmido } \\
\hline \multicolumn{6}{|l|}{ Radial } \\
\hline $\mathrm{PVA}_{\mathrm{c}}(1)$ & 30 & 0,43 & 2,271 & 1,356 & $70,68 \mathrm{a}$ \\
\hline EPI (2) & 30 & 0,50 & 2,271 & 2,012 & 68,85 a \\
\hline PU (3) & 30 & 1,00 & 2,271 & 2,912 & $77,98 \mathrm{a}$ \\
\hline \multicolumn{6}{|l|}{ Tangencial } \\
\hline $\mathrm{PVA}_{\mathrm{c}}(4)$ & 30 & 1,00 & 2,271 & 4,025 & 69,76 a \\
\hline EPI (5) & 30 & 17,33 & 2,271 & 19,771 & $138,53 \mathrm{~b}$ \\
\hline PU (6) & 30 & 11,80 & 2,271 & 22,530 & $117,18 \mathrm{c}$ \\
\hline $\mathrm{T}_{\text {calculado }}$ & & & & & 75,2259 \\
\hline P-valor & & & & & 0,00000 \\
\hline
\end{tabular}

* Classificação das médias conforme Teste de Kruskal-Wallis.

paramétrico de Kruskal-Wallis, testando a hipótese de nulidade onde todas as medianas fossem iguais entre os tratamentos. Como resultado, a hipótese de nulidade foi rejeitada $(\mathrm{P}$-valor $<0,05)$ para ambos os condicionamentos aplicados (a seco e a úmido), ou seja, existe diferença significativa dos tipos de adesivos e orientação de colagem na falha da madeira.

Os resultados de classificação das médias (Tabela 3) demonstraram que a falha da madeira em cisalhamento a seco do adesivo EPI colado no sentido radial foi superior aos demais tratamentos analisados e difere significativamente da falha na madeira do adesivo $\mathrm{PVA}_{\mathrm{c}} \mathrm{e}$

Cerne, Lavras, v. 19, n. 4, p. 613-619, out./dez. 2013 
do adesivo PU colados no sentido radial e tangencial e do adesivo EPI colado no sentido tangencial. Os resultados da falha da madeira em cisalhamento à úmido, demonstraram que o adesivo EPI e o adesivo PU colados no sentido tangencial foram superiores aos demais tratamentos analisados, diferindo significativamente do adesivo PVA em ambas as orientações de colagem, do adesivo EPI e do adesivo PU no sentido radial.

\section{CONCLUSÕES}

Com base nos resultados, foi observada a influência significativa do tipo de adesivo e da orientação de colagem na resistência da linha de cola da madeira de Pinus taeda. Em condicionamento a seco, os painéis de madeira de Pinus taeda colados no sentido radial apresentaram valores médios superiores aos colados no sentido tangencial para os três tipos de adesivos analisados. Em condicionamento a úmido, não foi possível observar influência significativa da orientação de colagem, porém a interação foi significativa entre os fatores adesivo e a orientação de colagem.

$\mathrm{O}$ adesivo PU apresentou os maiores valores médios de resistência ao cisalhamento nas etapas de condicionamento analisadas, diferindo estatisticamente do adesivo PVA $\mathrm{c}$ e do adesivo EPI quanto testado a seco e não diferindo dos demais adesivos quanto testado a úmido.

Em relação à falha na madeira, concluí-se que existe diferença estatística entre os tratamentos aplicados, sendo que o maior valor de falha na madeira foi observado após o ensaio de resistência ao cisalhamento a seco para $\mathrm{o}$ adesivo EPI colado no sentido radial. E no ensaio de resistência ao cisalhamento a úmido, o adesivo EPI e o adesivo PU colados no sentido tangencial apresentaram valores médios superiores de falha na madeira.

\section{AGRADECIMENTO}

Os autores agradecem à empresa National Starch $\&$ Chemical Industrial Ltda pelo fornecimento da matériaprima e apoio técnico.

\section{REFERÊNCIAS}

\author{
ASSOCIAÇÃO BRASILEIRA DA INDÚSTRIA DE \\ MADEIRA MECANICAMENTE PROCESSADA. \\ Estudo setorial 2007: indústria de madeira processada \\ mecanicamente, ano base 2006. Curitiba, 2007.
}

BANDEL, A. Adhesivos y tecnología del encolado en la industria de la madera. Milano: Rib\&s, 1991.

Cerne, Lavras, v. 19, n. 4, p. 613-619, out./dez. 2013
BURDURLU, E.; KILIÇ, Y.; ELI'BOL, G. C.; KILIÇ, M. Shear strength of calabrian pine (Pinus brutia Ten.) bonded with polyurethane and polyvinyl acetate adhesives. Journal of Applied Polymer Science, New York, v. 100, p. 4856-4867, 2006.

BUSTOS, C.; BEAUREGARD, R.; MOHAMMAD, M.; HERNÁNDEZ, R. E. Structural performance of finger-jointed black spruce wood lumber with different joint configurations. Forest Products Journal, Madison, v. 53, n. 9, p. 72-76, 2003.

CARNEIRO, A. de C. O.; VITAL, B. R.; PIMENTA, A. S.; MORI, F. A. Reatividade dos taninos da casca de Eucalyptus grandis para produção de adesivos. Cerne, Lavras, v. 7, n. 1, p. 1-9, 2001.

CONNER, A. H. Wood: adhesives: encyclopedia of materials: science and technology. New York: Elsevier Science, 2001.

EUROPEAN STANDARD. EN 204-01: classification of thermoplastic wood adhesives for non-structural applications. Brussels, 2001.

EUROPEAN STANDARD. prEN 205-02: adhesives: wood adhesives for non-structural applications: determination of tensile shear strength of lap joints. Brussels, 2002.

FOTSING, J. A. M.; ALEXIS, M. Strength of some wood adhesives used in Cameroon. International Journal of Adhesion \& Adhesives, Guildford, v. 23, p. 297-291, 2003.

FRIHART, C. R. Wood adhesion and adhesives: handbook of wood chemistry and wood composites. Boca Raton: CRC, 2005.

IWAKIRI, S.; KEINERT JUNIOR, S.; ALBUQUERQUE, C. E. C. de. Painéis de madeira reconstituída. Curitiba: FUPEF-UFPR, 2005.

KOLMANN, F. F. P.; KUENZI, E. W.; STAMM, A. J. Principles of wood science and technology: II., wood based material. New York: Springer-Verlag, 1975.

MITCHELL, P. H.; LEMASTER, R. L. Investigation of machine parameters on the surface quality in routing soft maple. Forest Products Journal, Madison, v. 52, n. 6, p. 85-90, 2002.

ÖZÇIFÇI, A.; YAPICI, F. Effects of machining method and grain orientation on the bonding strength of some wood species. Journal of Materials Processing Technology, Amsterdam, v. 202, n. 1/3, p. 353-358, June 2007. 
PAGEL, H. F.; LUCKMANN, E. R. A new isocyanate containing wood adhesive. Journal of Applied Polymer Science, New York, v. 40, p. 191-202, 1984.

VITAL, B. R.; MACIEL, A. da S.; DELLA LUCIA, R. M. Qualidade de juntas coladas com lâminas de madeira oriundas de três regiões do tronco de Eucalyptus grandis, Eucalyptus saligna e Pinus elliottii. Revista Árvore, Viçosa, MG, v. 30, n. 4, p. 637-644, jul./ago. 2006.

UYSAL, B. Bonding strength and dimensional stability of laminated veneer lumbers manufactured by using different adhesives after the steam test. International Journal of Adhesion \& Adhesives, Guildford, v. 25, p. 395-403, 2005.

Recebido: 29 de março de 2011; aceito: 24 de maio de 2013. 
